\title{
POSTER
}

\section{Les implants zygomatiques : indications, Technique de mise en charge immédiate, présentation de cas}

Romanet $\mathrm{I}^{1}$, Massereau $\mathrm{E}^{2}$, Tavitian $\mathrm{P}^{3}$, Richard $\mathrm{O}^{4}$, Catherine $\mathrm{JH}^{5}$

1,2,5 - Service de chirurgie orale Pavillon Dentaire Marseille

4 - chirurgien Maxillo facial et stomatologue Marseille clinique clairval

ivanromanet@hotmail.fr

Mots clés : Implant zygomatique, atrophie maxillaire, réhabilitation prothétique, mise en charge immédiate

Keywords : Zygomatic implants,maxillary atrophy, prosthetic rehabilitation, immediate load

La réhabilitation de l'os maxillaire atrophié est un challenge pour le chirurgien maxillo facial et le chirurgien oral. Chez la plupart des patient édentés totaux avec une atrophie maxillaire, le traitement conventionnel ne peut être réalisé du fait du déficit d'os alvéolaire aggravé par la pneumatisation du sinus maxillaire.

Conventionnelement, le déficit osseux est traité par des greffes d'apposition cortico-médullaire d'origine iliaque ou pariétale.

Cette procédure reconstructrice est complexe car elle nécessite plusieurs temps opératoires.

Les qualités de régénération de l'os zygomatique et la présence d'un os cortical très dense assurent une bonne stabilité primaire et favorisent l'ostéointégration des implants. En moyenne, la hauteur d'os disponible est de 14,1mm et la dimension antéro-postérieure de $20 \mathrm{~mm}$. Cet ancrage permet d'éviter la réalisation de greffes osseuses. La bonne stabilité primaire autorise le protocole de mise en charge immédiate qui entraine une diminution du temps de latence entre la chirurgie et la réhabilitation prothétique et, favorise une satisfaction rapide des patients, augmentant le confort et la qualité de vie du patient édenté total, moyennement ou fortement atrophié.

De nos jours, les implants zygomatiques permettent d'éviter les greffes osseuses multiples, un gain de temps majeur grace au protocole de mise en charge immédiate qui sera mise en évidence.

Le taux de survie élevé (supérieur à $90 \%$ ) et la faible incidence de complications rapportées, font des implants zygomatiques une bonne option de traitement pour la réhabilitation de maxillaires sévèrement atrophiés.

Références

(1) Cawood JL., Howell RA. A classification of the edentulous jaws. Int J Oral Maxillofac Surg. 1988;17:232-236.

(2) Bedrossian E., Sullivan RM., Malo P. Fixed-Prosthetic Implant Restoration of the Edentulous Maxilla : A Systematic Pretreatment Evaluation Method. J Oral Maxillofac Surg. 66:112-122, 2008.

(3) Bedrossian E. Rehabilitation of the Edentulous Maxilla with the Zygoma Concept : A 7-year Prospective Study. INT J ORAL MAXILLOFAC IMPLANTS 2010;25: 1213-12217.

This is an Open Access article distributed under the terms of the Creative Commons Attribution License 4.0, which permits unrestricted use, distribution, and reproduction in any medium, provided the original work is properly cited. 